\title{
Unusual presentation of endometriosis with massive ascites and recurrent pleural effusion: a case report and review of the literature
}

Received: 21 May 2006 / Accepted: 8 June 2006 / Published online: 22 July 2006

(C) Springer-Verlag Berlin / Heidelberg 2006

\begin{abstract}
A 31-year-old nulliparous woman presented to the respiratory physicians with a 3-month history of abdominal distension, weight loss, increasing shortness of breath and change in bowel habit. On examination she was frail and thin with signs of massive right-sided pleural effusion and ascites. This was confirmed on ultrasound scan and computed tomography (CT) scan which also revealed a simple left adnexal cyst. Tumour markers were negative. As the pleural effusion recurred after drainage, a laparotomy was done to exclude ovarian malignancy and this revealed grade 3 to 4 endometriosis. She declined medical or surgical management of her endometriosis despite recurring pleural effusions.
\end{abstract}

Keywords Endometriosis · Pleural effusion · Ascites · Dysmenorrhoea $\cdot$ Gonadotrophin-releasing hormone analogues (GnRHa)

\section{Case report}

A 31-year-old lady presented to the respiratory physicians with a 3-month history of abdominal distension, weight loss of 4 stone, increasing shortness of breath and change in bowel habit (on and off diarrhoea and constipation).

She was a nulligravid, never used contraception and had a regular menstrual cycle associated with dysmenorrhoea. She had never had a smear but had no other past medical or surgical history of note.

On examination she was frail and thin. Respiratory examination showed her to be tachypnoeic with the right chest stony dull to percussion. Abdominal examination revealed a striking ascites.

S. Mwenechanya $\cdot$ I. Beck $(\bowtie)$

Obstetrics and Gynaecology, Bradford Royal Infirmary,

Duckworth Lane,

Bradford, BD9 6RJ, UK

e-mail: Ian.Beck@bradfordhospitals.nhs.uk

Tel.: +44-1274542200

Fax: +44-1274366690
Chest X-ray confirmed a large right pleural effusion causing mediastinal shift to the left. The left lung was clear.

Ultrasound of the pelvis and abdomen showed large volume ascites; the liver was normal in size but of coarse bright echogenicity. Both kidneys were enlarged with multiple cysts; the uterus was a little enlarged with fibroids and a $11 \times 7 \times 7 \mathrm{~cm}$ apparently simple cyst on the left side of the pelvis. This was confirmed on computed tomography (CT) scan. No lymph nodes were seen.

Full blood count, clotting, urea and electrolytes, and liver function tests were normal. CEA was less than 1, CA15.3 was 21 , CA19.9 was 8 and CA125 was 20 . HIV (human immunodeficiency virus) test was negative

She had a chest drain inserted the day of admission which drained more than 51 of frank blood-stained fluid in $24 \mathrm{~h}$ and continued to drain large amounts over the following days. Repeat chest X-ray showed full lung expansion with minimal pleural thickening.

Four days later she developed a pneumothorax which resolved over the following few days.

Unfortunately the pleural fluid was too heavily blood stained for cytology. Because of the ovarian cyst noted on CT scan she was referred to the gynaecological oncologist. A pleural biopsy was suggested to aid diagnosis as the ovarian cyst appeared simple on scan with no features suggestive of ovarian malignancy and normal tumour markers.

She was discharged home 11 days after her admission, her symptoms having improved after drainage of the pleural effusion.

Three months later she was readmitted with right pleural effusion recurrence and had a chest drain reinserted. The chest surgeons thought the diagnosis was Meigs' syndrome and re-referred her to the gynaecologists.

A laparotomy was done where 11 of ascites was drained, and the uterus tubes and ovaries were all bound down in the pelvis with adhesions. Grade 3 to 4 endometriosis was found. The ovarian cyst was aspirated and fluid sent for cytology. The peritoneal lesions were biopsied. The omentum and liver looked normal. 
Histology confirmed endometriosis with no evidence of malignancy. Ovarian fluid cytology showed no evidence of malignancy.

Three months later the pleural effusion recurred and a long-term chest drain was inserted. A pleural biopsy was taken which showed pleural endometriosis and pleurodesis was done at the same time.

At the 2-month follow-up she was well with no evidence of pleural effusion recurrence. She declined medical or surgical management of her endometriosis.

\section{Discussion}

Endometriosis is one of the common conditions seen in gynaecology clinics among women of childbearing age. It usually presents with dysmenorrhoea, pelvic pain, dyspareunia or infertility.

We have described a case of endometriosis which presented unusually with massive pleural effusion and ascites mimicking malignant disease. Ultrasound and CT findings of abnormal looking liver and kidneys with only a simple looking ovarian cyst made diagnosis of a gynaecological cause for the ascites and effusion less likely and one of advanced metastatic disease more likely.

Only at laparotomy to exclude ovarian malignancy was the diagnosis of endometriosis made and that of pleural endometriosis confirmed at pleural biopsy.

In 1954 Brews described an unusual case of ascites associated with endometriosis [1]. There have been 32 cases described in the English literature of endometriosis associated with ascites [2]. The presentation of endometriosis with both pleural effusion and ascites as in the above-described case is even rarer with only 15 cases described in the English literature. The pleural effusion is mostly right sided with a few cases having bilateral effusions [3].

There are two cases in the literature of endometriosis associated with pericardial effusion as well as pleural effusion and ascites $[4,5]$. In one case of a multiparous woman the effusions resolved after total abdominal hysterectomy and salpingo-oophorectomy (TAH and BSO) [5]; the other case had recurrent effusions despite pleurectomy, left oophorectomy (right ovary left in) and treatment with gonadotrophin-releasing hormone analogues (GnRHa) [4].

Two English abstracts from French papers have described pregnancy after treatment of endometriosis associated with ascites. One conceived with in vitro fertilization (IVF) after treatment with GnRHa. The ascites did not progress during pregnancy but recurred after delivery which was at full term [6]. The second case had recurrent pleural effusion after stopping treatment with $\mathrm{GnRHa}$ and was found to be pregnant when she presented with the recurrent pleural effusion and ascites [7].

The cases described mainly present with a few months history of shortness of breath, abdominal distension (84\%), pelvic mass (50\%), dysmenorrhoea (38\%), pleural effusion $(34 \%)$,weight loss $(34 \%)$, reduced appetite $(22 \%)$ and change in bowel habit. All the above findings are more suggestive of malignant ovarian disease than endometriosis especially if CA125 is also elevated as was found in some of these cases [8].

The majority of these women are black and nulliparous and of childbearing age. Reported age ranged from 19 to 65 years (median age 29) [2]. Of the women described in the literature with ascites, $85 \%$ of those whose parity was recorded were nulliparous and of those whose race was reported $81 \%$ were black. The majority of the pleural effusions are right sided with a few having bilateral effusions. The ascites is usually massive with amounts ranging from 1.5 to 101 and described as serosanguineous, sanguineous or brown.

Diagnosis is usually difficult due to the unusual presentation especially mimicking malignancy with the patients undergoing extensive investigations. These patients may therefore present to respiratory physicians, gastroenterologists or gynaecologists. The differential diagnosis includes:

\section{- Ovarian cancer \\ - Meigs' syndrome \\ - Liver tumour or cirrhosis \\ - Tuberculosis \\ - Renal or cardiac disease \\ - Portal vein thrombosis}

The ascites is usually an exudate [2] with reactive mesothelial cells found on cytology giving rise to the theory that blood from ruptured endometriotic cysts causes peritoneal irritation leading to the exudative ascites $[8,3$, 9-13].

It is postulated that the pleural effusion is either due to trans-diaphragmatic transfer of ascetic fluid or leakage of the overloaded lymphatic system or even as recorded in one case [14] through diaphragmatic perforations $[2,4,9$, 13]. Why this only happens in some patients and not in others is not clear. Indeed the presence of endometriosis in the pleura of some of these patients may mean that this is a separate exudative process from pleural irritation by pleural endometriosis.

Treating these patients can be difficult as they are usually of childbearing age and nulliparous and so wish to preserve their fertility. Nearly all cases underwent diagnostic surgery in the first instance to exclude malignancy. As well as massive ascites dense pelvic adhesions are found in all cases at laparotomy.

Like all cases of endometriosis medical management with combined oral contraceptive pill, danazol, DepoProvera [15], leuprolide acetate and norethindrone acetate has been described as effective in preventing recurrences but ovarian ablation either surgically or by radiotherapy (early cases by Brews and Bernstein) has been needed for resistant cases.

Most patients are managed with GnRHa as first line [2, 16] but a few have recurrent ascites not responding to medical management and require bilateral oophorectomy.

The pleural effusion is usually recurrent requiring thoracotomy and pleurodesis or pleurectomy to prevent 
recurrence especially if fertility-preserving treatment is used for the endometriosis $[4,3,17,18]$.

\section{Summary}

Endometriosis associated with ascites is rare. Endometriosis associated with both ascites and pleural effusion is even rarer. As it presents with symptoms similar to metastatic ovarian malignancy both gynaecologists and other specialists like respiratory physicians, thoracic surgeons and gastroenterologists need to be aware of it as a differential diagnosis especially in women of childbearing age. Conservative medical management for symptomatic control with endocrine therapy like GnRH analogues, Provera, danazol and norethindrone has an important place as most of these women are nulliparous and wish to preserve their fertility. However, for recurrent cases ovarian ablation by either oophorectomy or irradiation as in the earlier cases seems to be the only way to get permanent cure.

\section{References}

1. Brews A (1954) Endometriosis including endometriosis of the diaphragm and Meig's syndrome. Proc R Soc Med 47:461

2. Dias CC, Jurandyr M, Andrade JM, Ferriani RA, Marcia G, Villanova MG, Meirelles RS (2000) Hemorrhagic ascites associated with endometriosis. A case report. J Reprod Med 45:688-690

3. Bhojawala J, Heller DS, Cracchiolo B, Sama J (2000) Endometriosis presenting as bloody pleural effusion and ascites-report of a case and review of the literature. Arch Gynecol Obstet 264:39-41
4. Flanagan KL, Barnes NC (1996) Pleural fluid accumulation due to intra-abdominal endometriosis: a case report and review of the literature. Thorax 51:1062-2063

5. Francis M, Oluyemi O, Bodero OO, Borowsky M, Lee Y, Abulafia O (2003) Pericardial effusion, right-sided pleural effusion and ascites associated with stage IV endometriosis. A case report. J Reprod Med 48:463-465

6. Ekoukou D, Guilherme R, Desligneres S, Rotten D (2005) Endometriosis with massive hemorrhagic ascites: a case report and review of the literature (in French). J Gynecol Obstet Biol Reprod (Paris) 34(4):351-359

7. Fortier D, Dedecker F, Gabriele M, Graesslin O, Barau G (2005) Endometriosis with ascites and pleural effusion: a case report (in French). Gynecol Obstet Fertil 33(7-8):508-510

8. Spitzer M, Benjamin F (1995) Ascites due to endometriosis. Obstet Gynecol Surv 50:628-631

9. Myers TJ, Arena B, Granai CO (1995) Pelvic endometriosis mimicking advanced ovarian cancer: presentation with pleural effusion, ascites and elevated serum CA125 level (case report). Am J Obstet Gynecol 173(3):966-967

10. Bernstein JS, Perlow V, Brenner JJ (1961) Massive ascites due to endometriosis. Am J Dig Dis 6:1

11. Irani S, Atkinson L, Cabaniss C et al (1976) Pleural peritoneal endometriosis. Obstet Gynecol 47(Suppl):72

12. Jenks JE, Artman LE, Hoskins WJ et al (1984) Endometriosis with ascites. Obstet Gynecol 63:75-77

13. Chen FF, Chow NH, Chou CY, Lin MF (1992) Haemorrhagic ascites associated with endometriosis: a rare clinical presentation. J Gynecol Surg 8:43-47

14. Karpel JP, Appel D, Merav A (1985) Pulmonary endometriosis. Lung 163:151-159

15. Charran D, Roopnarinesingh S (1993) Hemothorax and ascites associated with endometriosis. West Indian Med J 42:40-41

16. Shek Y, De-Lia JE, Patillo RA (1995) Endometriosis with pleural effusion and ascites. Report of a case treated with nafarelin acetate. J Reprod Med 40(7):540-542

17. Ziedalski TM, Sankaranarayanan V, Chitkara RK (2004) Thoracic endometriosis: a case report and literature review. J Thorac Cardiovasc Surg 127:1513-1514

18. Moffatt SD, Mitchell JD (2002) Case report; massive pleural endometriosis. Eur J Cardiothorac Surg 22:321-323 